\title{
A Model with Electric Fields for the Inclusion of Mutual Coupling Effects in the MIMO Channel
}

\author{
Celal Alp Tunc, Erdinc Irci, Onur Bakir, \\ Defne Aktas, Vakur B. Ertürk and Ayhan Altintas \\ Dept. of Electrical and Electronics Engineering, Bilkent University, \\ Bilkent, Ankara, Turkey, TR-06800, Email: celal@ee.bilkent.edu.tr
}

\section{Introduction}

Multiple Input Multiple Output (MIMO) wireless communication systems have been a focus of interest, due to their ability to increase the capacity in rich scattering environments by using multi-element antenna arrays both at the transmitter and the receiver sides $[1,2]$. However, when dealing with multi-element antenna arrays, effects of mutual coupling among the array elements become significant and should be included in the channel matrix properly. These effects were included in the MIMO channel matrix mainly for free standing linear arrays (FSLA) of uniform thin-wire dipole antennas using coupling matrices obtained from the mutual interaction matrix and terminations in $[3,4]$. These matrices reduce to the identity matrix when the interactions are ignored, because of the scaling factors related with termination impedances. Unfortunately, if a comparison among various array types is desired, this approach fails since it gives the same channel capacity regardless of the type of elements in an array. Therefore, in this paper we propose a partially stochastic full-wave electromagnetic model with electric fields (MEF), to evaluate the MIMO channel matrix accurately with and/or without including effects of mutual coupling. Effects of mutual interactions among the array elements through space and surface waves (when printed arrays are considered) are included in the channel matrix using a full-wave hybrid method of moments (MoM)/Green's function technique. The stochastic part of the model comes from a local cluster of uniformly distributed scatterers. Consequently, the proposed method is exact except the scatterer scenario, thus, besides achieving the accuracy to be used as a benchmark solution for other approaches, comparisons can be made among any kind of arrays. Throughout this paper, an $e^{j w t}$ time convention is used and suppressed from the expressions.

\section{Channel Model with Electric Fields}

The scattering environment, adopted from [3], is a two dimensional (2D), singlebounce geometric model. It assumes a local cluster of scatterers around the transmitter array (TX) and the receiver array (RX). The local cluster is a disk of radius $R_{D}$ including $S$ uniformly distributed scatterers. Assuming flat fading, the received signal vector, $\bar{v}^{r x}$, can be written in terms of the transmitted one, $\bar{v}^{t x}$, and the additive white Gaussian noise vector, $\bar{n}$, with zero mean independent identically distributed elements with unit variance as: $\bar{v}^{r x}=\mathbf{H} \bar{v}^{t x}+\bar{n} ; \mathbf{H}$ denotes the $R \times T$ channel matrix, where $R$ and $T$ are the number of antenna elements in receiver and transmitter arrays, respectively. Assuming the channel knowledge only at the receiver side, the maximum amount of data to be transmitted reliably (i.e., the Shannon capacity), can be evaluated as $\mathcal{C}=\log _{2}\left(\left|\mathbf{I}+P_{T} \mathbf{H H}^{*} / T\right|\right)$, where $I$ is 
the $R \times R$ identity matrix, $|$.$| is the matrix determinant, P_{T}=E\left[\bar{v}^{t x *} \bar{v}^{t x}\right]$ is the total transmitted power with (.)* and $E[$.$] denoting the conjugate transpose and$ expectation operations, respectively. Mutual coupling effects were included into the channel model for uniform arrays of side-by-side free standing linear dipoles (FSLA) in $[3,4]$ using the coupling matrices for RX and TX which are obtained from mutual impedance matrices. Comparing different array types, such as FSLA versus printed linear dipole arrays (PLDA), the method fails, since model is based on the phase differences due to scatterers and spatial properties of antenna elements, and will yield exactly the same channel matrix regardless of the type of elements in an array with the same interelement spacing values for no mutual coupling cases.

Here, we propose a full-wave electromagnetic model with the same scatterer scenario. In order to involve the electric fields, the channel matrix $\mathbf{H}$ is splitted into two as $\mathbf{H}_{1}$ and $\mathbf{H}_{2} . \mathbf{H}_{1}$ is the $S \times T$ transmission link matrix relating TX to scatterers; whereas $\mathbf{H}_{2}(R \times S)$ is the one linking scatterers to RX. The overall channel matrix, $\mathbf{H}$ can then be expressed as: $\mathbf{H}=\mathbf{H}_{2} \mathbf{H}_{1}$. Using electric fields, the $(p, n)$ th entry of $\mathbf{H}_{1}$ is written as: $h_{p n}^{(1)}=E^{t x}\left(\bar{r}_{n p}\right), v_{n}^{t x}=1 \mathrm{~V}, v_{i \neq n}^{t x}=0$, where $E^{t x}\left(\bar{r}_{n p}\right)$ denotes the electric field generated by transmitter array, impinging on the $p$ th scatterer, when $n$th TX antenna is activated. The incident energy is scattered at the scatterers. Then, treating each scatterer as an isotropic radiator, the $(m, p)$ th entry of $\mathbf{H}_{2}$ is expressed as $h_{m p}^{(2)}=\alpha_{p} E^{r x}\left(\bar{r}_{p m}\right), v_{m}^{r x}=1 \mathrm{~V}, v_{q \neq m}^{r x}=0$ due to the reciprocity principle. In order to obtain the entries of mutual coupling included $\mathbf{H}_{1}$, the following procedure of the MEF is used:

i. Evaluate $\mathbf{Z}_{T}$.

ii. Start with $n=1$.

iii. Activate $n$th TX element $\left(v_{n}^{t x}=1 \mathrm{~V}, v_{j \neq n}^{t x}=0\right)$.

iv. Calculate the current vector, $\bar{i}^{t x}=\left(\mathbf{Z}_{T}+Z_{S} \mathbf{I}\right)^{-1} \bar{v}^{t x}$.

v. Find

$$
h_{p n}^{(1)}=E^{t x}\left(\bar{r}_{n p}\right)=\sum_{t=1}^{T} E\left(i_{t}, p\right)
$$

vi. Increase $n$, and go to (iii).

In $(1), E\left(i_{t}, p\right)$ is the electric field due to the current $i_{t}$ on the $t$ th element in the transmitter array impinged on $p$ th scatterer, i.e.,

$$
E\left(i_{t}, p\right)=\int_{S_{t}} J_{t}\left(\bar{r}^{\prime}\right) G\left(\bar{r}_{p}, \bar{r}^{\prime}\right) d s^{\prime}
$$

for $j, n, t=1,2, \ldots, T$, where $\int_{S_{t}}(.) d s^{\prime}$ is the integral over the $t$ th element, $J_{t}$ is the current density on the same element due to $i_{t},\left(\bar{r}_{p}, \bar{r}^{\prime}\right)$ is the position vector pair for the $p$ th scatterer and th antenna element, and finally $G\left(\bar{r}_{p}, \bar{r}^{\prime}\right)$ denotes the 

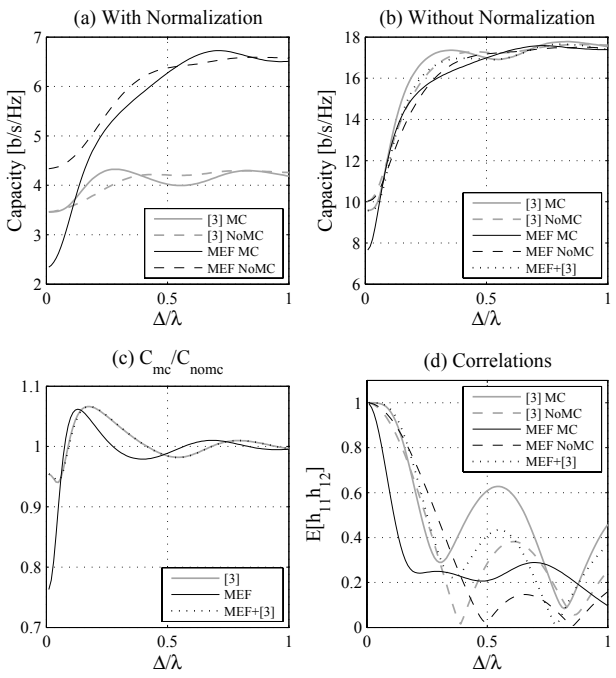

Figure 1: MIMO capacity comparison of MEF and [3] versus interelement spacing (a) with and (b) without normalization of the channel matrix for FSLA. Mutual coupling included. (c) Comparison of mutual coupling inclusion methods, MEF versus [3] (d) Correlation comparison of MEF and [3] versus interelement spacing. $S=100, R_{D}=200 \lambda$.

Green's function (A similar procedure can be followed to obtain the entries of $\mathbf{H}_{2}$, by using RX array properties). Thus, in steps (iv)-(v), the mutual interactions among the array elements through space and surface waves (when printed arrays are considered) are included in the channel matrix using a full-wave hybrid method of moments (MoM)/Green's function technique. Besides, effect of the termination impedance on MIMO channel capacity is examined as well. Hence, the proposed model is exact (except the scatterer scenario) and can be used as a benchmark solution for validation of other channel models. Also, it can be used for arrays of any kinds of antenna elements.

\section{Numerical Results}

MIMO performances of linear arrays of free standing (FSLA) dipole elements are investigated in terms of mean channel capacity, received SNR and correlations. The channel model we propose (depicted as MEF), and the one in [3] are compared for the cases with and without mutual coupling (represented by MC and NoMC in the figures, respectively). Transmit and receive arrays are assumed to be identical linear arrays, located $300 \lambda$ away from each other in a broadside manner and formed by $R=T=2$ uniform dipoles, where each of them is separated by a distance of $\Delta$. Results are generated for different $\Delta$ values between $0.01 \lambda$ and $\lambda$. Mean capacity results are obtained by averaging the MIMO channel capacity over $N_{R}=$ 1000 channel realizations. FSLA are considered to be composed of thin wire dipole elements of $\lambda / 2$ height and $\lambda / 200$ radius. An equalization process is considered for MEF with fixed transmit power in order to have a fair comparison. First, the single input single output (SISO) capacity for [3] with normalization is calculated 
by $\mathcal{C}_{\text {siso }}=\log _{2}(1+S N R)$ for $S N R=10 \mathrm{~dB}$, and then, required $P_{T}$ of MEF for the same $\mathcal{C}_{\text {siso }}$ is obtained around $99 \mathrm{~dB}$ and fixed for all $\Delta$ values. Results are illustrated in Fig. 1 (a) both for [3] and MEF with and without mutual coupling. [3] differs so much from the MEF, because of the normalization of $\mathbf{H}$. Removing the normalization, SISO capacity of [3] alters to $\mathcal{C}_{\text {siso }}=E\left[\log _{2}\left(1+S N R\left|h_{11}\right|^{2}\right)\right]$. The required $P_{T}$ of $\mathrm{MEF}$ for this $\mathcal{C}_{\text {siso }}$ is calculated to be around $117 \mathrm{~dB}$ and fixed. Results become comparable when the normalization is avoided as shown in Fig. 1 (b). Normalization cancels some channel characteristics, and should be removed in order to have a correct comparison. In Fig. 1 (b) effect of the mutual coupling on the MIMO capacity is given by plotting the mean capacities of MEF and [3] with and without coupling. Also, results of a hybrid version of two models, in which without coupling case is obtained using MEF and mutual coupling effects are included by coupling matrices of [3], is shown. Further investigation on coupling inclusion techniques is done by simply plotting $\mathcal{C}_{m c} / \mathcal{C}_{\text {nomc }}$ in Fig 1 (c). Results clearly show that, the two inclusion models are not the same. Finally, channel correlation (i.e., $E\left[h_{11} h_{12}\right]$ ) is illustrated in Fig. 1 (d), for all the models with and without mutual coupling. Optimal values emerge around the correlation minima for [3] and MEF + [3]. However, this is not the case for MEF. [3] yields pretty different optimum distances from MEF when mutual coupling is included.

\section{Conclusion}

A model with electric fields based on a hybrid MoM/Green's function technique is proposed and used in order to evaluate the mutual coupling included MIMO channel matrix. The accuracy of the method in [3] is tested using our model with electric fields (MEF) as a benchmark solution.

\section{Acknowledgment}

This work is supported by the Turkish Scientific and Technical Research Agency (TÜBİTAK) under Grants EEEAG-106E081, EEEAG-104E044, EEEAG-105E065. The work has been also supported in part by the European Union Network of Excellence (NoE) NEWCOM, under the 6th Framework Programme.

\section{References}

[1] I. E. Telatar, "Capacity of multi-antenna gaussian channels," Europ. Trans. Telecommun., vol. 10, pp. 585-595, Nov. 1999.

[2] G. J. Foschini and M. J. Gans, "On limits of wireless communications in a fading environment when using multiple antennas," Wireless Personal Commun., vol. 6, pp. 311-335, Mar. 1998.

[3] T. Svantesson and A. Ranheim, "Mutual coupling effects on the capacity of multielement antenna systems," in Proc. IEEE Int. Conf. Acoustics, Speech, and Signal Processing (ICASSP'01), Salt Lake City, UT, May 2001, pp. 2485-2488.

[4] R. Janaswamy, "Effect of element mutual coupling on the capacity of fixed length linear arrays," IEEE Antennas Wireless Propagat. Lett., vol. 1, no. 1, pp. 157-160, 2002. 